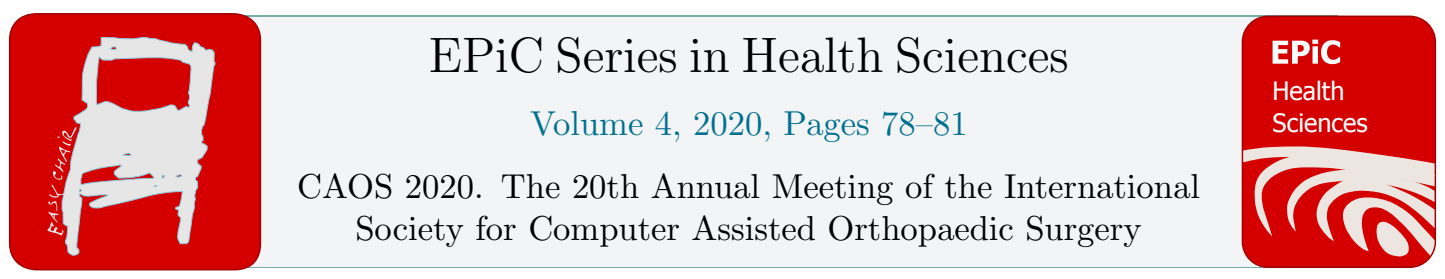

\title{
Projector-Based Augmented Reality System for Computer Assisted Orthopaedic Surgery
}

\author{
Yuan Gao, Le Xie, and Guoyan Zheng \\ Institute of Medical Robotics, School of Biomedical Engineering, \\ Shanghai Jiao Tong University, Shanghai, China \\ gao.yuan@sjtu.edu.cn, guoyan.zhengesjtu.edu.cn
}

\begin{abstract}
This paper presents a projector-based augmented reality (AR) system for ComputerAssisted Orthopaedic Surgery (CAOS). After calibration, our AR system allows for projection of not only the virtual model directly on the surface of the target organ to create an augmented reality but also important clinical information such as distance and angular deviations from a surgical plan, which are important for various computer-assisted surgical procedures such as trajectory drilling and fracture reduction. The feasibility and accuracy of the system is experimentally validated on a 3D printed phantom model with pyramid shape, a dry goat bone and an in vitro pig leg. An average projection distance error of $1.03 \pm 0.58 \mathrm{~mm}$ and an average drill alignment error of $1.17 \pm 0.43^{\circ}$ were found. The results demonstrate the efficacy of the proposed AR system.
\end{abstract}

\section{Introduction}

Augmented Reality (AR)-based surgical navigation systems draw more and more attentions in Computer-Assisted Orthopaedic Surgery (CAOS) community. There exist various ways to overlay the pre-operative surgical plan onto the intra-operative surgical scenario. AR could be one of the solutions. AR is defined as an advanced user interface that combines supplemental information with the realworld environment [1] and has made a great breakthrough over the last decades. This technology has been used in entertainment, military, manufacturing and medical fields. In medical field, AR is being used for training, planning, and intraoperative guidance. AR has been used to improve surgeons' performance during surgical procedures by overlaying image data and surgical planning onto the intraoperative surgical scenario. The AR system could be achieved with various means: head-mounted display (HMD) [2], portable LCD screen with a digital camera behind [3], optical see-through Microsoft Hololens [4], or projector-based AR system [5]. Comparing to other means, the projector-based AR systems generate less burden to the surgeon. In this paper, we developed a projector-based AR system and evaluated the performance of the system for surgical guidance. 

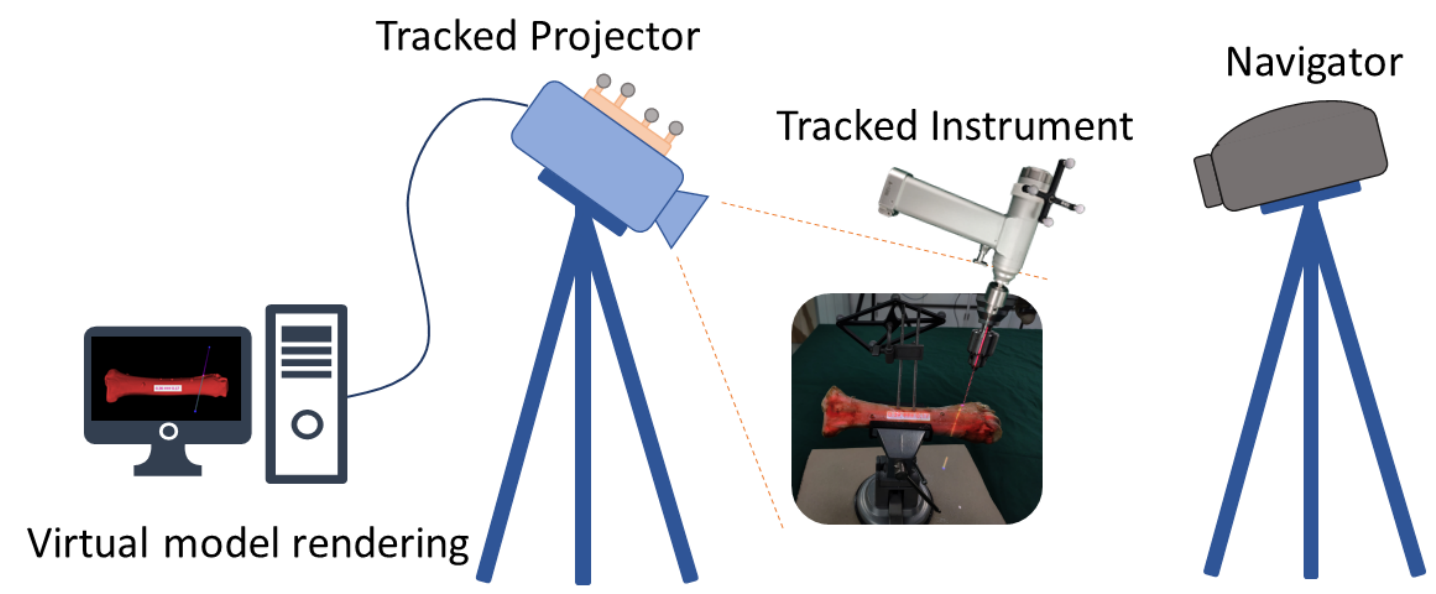

Figure 1. Overall setup of the projector-based AR system

\section{Materials and Methods}

The overall setup of our projector-based AR system is shown in Figure 1, where the projector (JmGo, China), the surgical drill (A-1200, Wuyang Medical Equiment Inc., Hefei, China), and patient's reference base are tracked by Polaris Vega camera (Northern Digital Inc., Ontario, Canada).

The projector is calibrated using a checkboard with known pattern, where the 2D pixel coordinates of all checkboard corners are known by design. More specifically, we render the checkboard on computer screen which is then projected onto a flat plate using the projector. The $3 \mathrm{D}$ coordinates of the projected checkboard corners are obtained by digitization using a tracked probe. Based on the 2D pixel coordinates and the associated 3D coordinates of the projected checkboard corners when the flat plate are placed in different locations and orientations, we can calibrate the projector to get its intrinsic and extrinsic parameters, which then allow us to create a virtual rendering environment that is aligned with the projector's optical system.

As soon as any virtual model is registered into the patient's reference base, it can be rendered using the virtual rendering environment such that in the patient's reference space the projected virtual image is aligned with the in-situ environment.

\section{Experimental Setup and Results}

We conducted validation experiments using a 3D printed phantom model with pyramid shape, a dry goat bone and an in vitro pig leg as shown in Figure 2. The virtual model of the 3D printed phantom model was obtained by computer aided design while the virtual models of both the dry goat bone and the in vitro pig leg were obtained from CT images.

The 3D printed phantom pyramid as shown in Figure 2-(a) has a set of pre-defined indents, which are designed to evaluate the projection accuracy by computing the distance between the projected indents and the indents on the phantom pyramid. The in vitro pig leg as shown in Figure 2-(b) was designed to qualitatively demonstrate the projection of the underlying bony structures on the skin of the pig leg. Finally, we used the dry goat bone to show the trajectory drilling accuracy when guided by our 
projector-based AR system. As shown in Figure 2-(c) and Figure 2-(d), our projector-based AR system projects not only the virtual bone models and the instrument axis onto the surface of the target anatomy but also the real-time linear and angular measurements, which can significantly help the surgeon to achieve accurate trajectory drilling. In total, we drilled 6 trajectories. Drilling accuracy was evaluated by comparing the drilled trajectories and the planned ones.

For the $3 \mathrm{D}$ printed phantom study, an mean projection error of $1.025 \pm 0.583 \mathrm{~mm}$ was found. For the dry goat bone study, an average drill alignment error of $1.17 \pm 0.43^{\circ}$ were found.

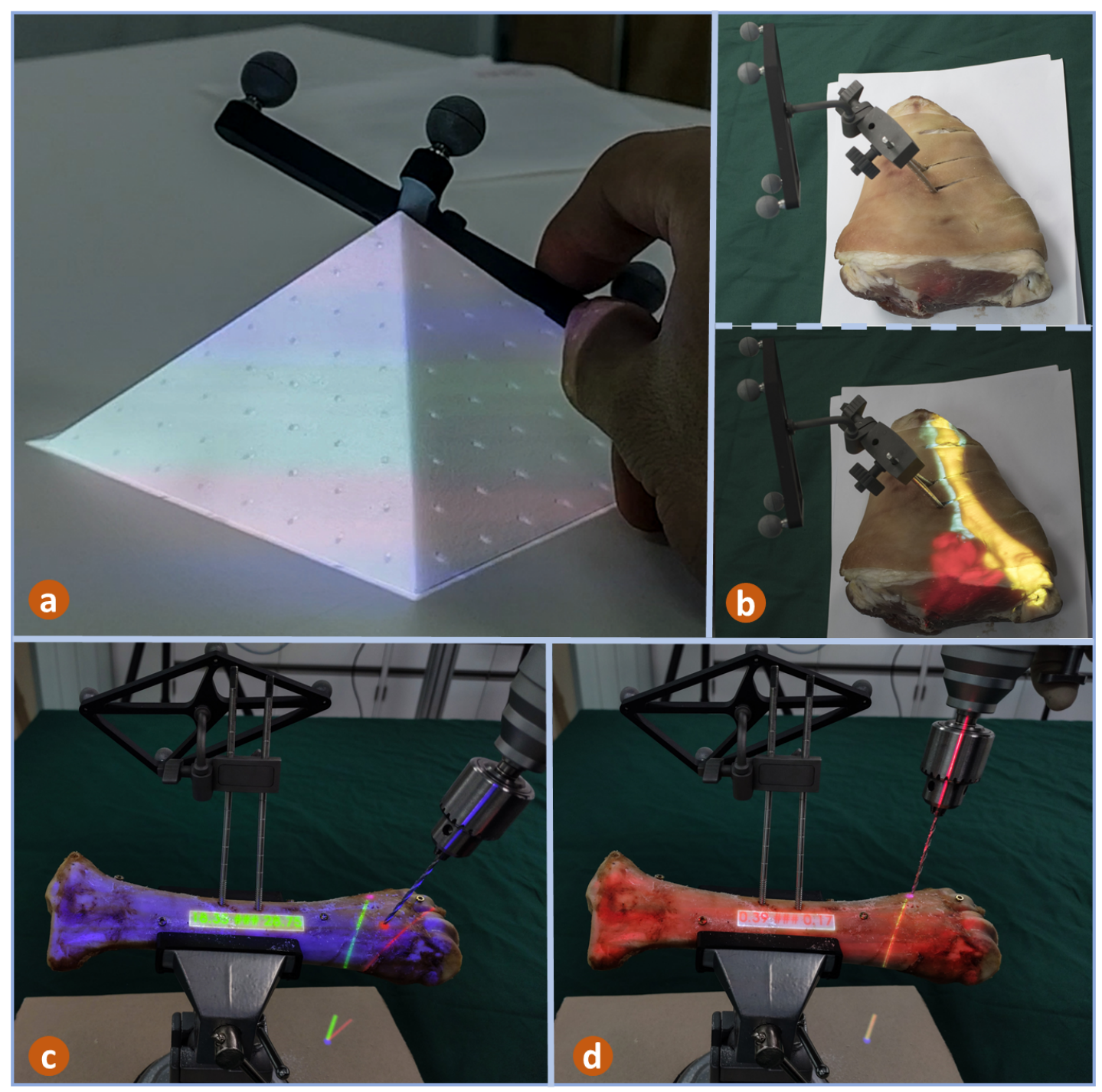

Figure 2. Experimental setup. (a) when the 3D printed phantom pyramid was used; (b) when the in vitro pig leg was used; (c) and (d) when the dry goat bone was used for trajectory drilling. 


\section{Discussions and Conclusions}

In computer-assisted orthopaedic surgery, the conventional navigation systems display real-time navigation information on a monitor, which is not optimal. Specifically, during surgical intervention, the surgeon has to shift eyes around the monitor and the patient, which may cause the loss of precision and increase the risk [6]. AR technique could be one of the solutions. In comparison with other types of AR systems such as head-mounted display (HMD) [2], portable LCD screen with a digital camera behind [3], and optical see-through Microsoft Hololens [4], projector-based AR systems can lead to increased performance and reduced cognitive load, which are confirmed by a recent study [7]. In this paper, we developed and validated a projector-based AR system for computer-assisted orthopaedic surgery. The difference between our system and other projector-based AR systems [5] lies in that our system can overlay not only the virtual models onto the in-situ surgical scenario but also important clinical information such as distance and angular measurements as shown in Figure 2-(c) and 2-(d), which can significantly help the surgeon to achieve more accurate operations. The experimental results demonstrate the efficacy of the proposed system.

\section{References}

[1] M. Blackwell, F. Morgan, and A. M. DiGioia III (1998), Augmented reality and its future in orthopaedics, Clinical Orthopaedics and Related Research, vol. 354, pp. 111-122

[2] C. Bichlmeier, B. Ockert, O. Kutter, M. Rustaee, S. M. Heining, and N. Navab (2008), The visible korean human phantom: Realistic test \& development environments for medical augmented reality, in International Workshop on Augmented environments for Medical Imaging including Augmented Reality in Computer-aided Surgery (AMI-ARCS 2008), New York, USA

[3] R. A. Mischkowski, M. J. Zinser, A. C. Kubler, B. Krug, U. Seifert, and J. E. Zoller (2006), Application of an augmented reality tool for maxillary positioning in orthognathic surgery-a feasibility study, J Craniomaxillofac Surg, vol. 34, no. 8, pp. 478-83.

[4] Y Zuo, T Jiang, J Dou, et al. (2020), A Novel Evaluation Model for a Mixed-Reality Surgical Navigation System: Where Microsoft HoloLens Meets the Operating Room, Surg Innov. in press.

[5] R. Wen, C. K. Chui, S. H. Ong, K. B. Lim, S. K. Chang (2013), Projection-based visual guidance for robot-aided RF needle insertion, Int J Comput Assist Radiol Surg, vol. 8, no. 6, pp. 1015-1025

[6] JR Wu, ML Wang, KC Liu, MH Hu, PY Lee (2014), Real-time advanced spinal surgery via visible patient model and augmented reality system, Comput Methods Programs Biomed,113(3):869881

[7] J Baumeister, SY Ssin, NAM EiSayed, J Dorrian, DP Webb, et al. (2017), Cognitive Cost of Using Augmented Reality Displays, IEEE Trans Vis Comput Graph.;23(11):2378-2388. 